\title{
Interval-based Solving of Hybrid Constraint Systems *
}

\author{
Daisuke Ishii ${ }^{*}$ Kazunori Ueda ${ }^{*, * *}$ Hiroshi Hosobe ${ }^{* *}$ \\ Alexandre Goldsztejn ${ }^{* * *}$ \\ * Dept. of Computer Science, Waseda University, 3-4-1, Okubo, \\ Shinjuku-ku, Tokyo 169-8555, Japan \\ (e-mail: $\{$ ishii, ueda\}@ueda.info.waseda.ac.jp). \\ ** National Institute of Informatics, 2-1-2, Hitotsubashi, Chiyoda-ku, \\ Tokyo 101-8430, Japan (e-mail: hosobe@nii.ac.jp) \\ *** LINA, Université de Nantes, 2, Rue de la Houssinnière, BP 92208, \\ F-44322 Nantes Cedex 3, France \\ (e-mail: alexandre.goldsztejn@univ-nantes.fr)
}

\begin{abstract}
An approach to reliable modeling, simulation and verification of hybrid systems is interval arithmetic, which guarantees that a set of intervals narrower than specified size encloses the solution. Interval-based computation of hybrid systems is often difficult, especially when the systems are described by nonlinear ordinary differential equations (ODEs) and nonlinear algebraic equations. We formulate the problem of detecting a discrete change in hybrid systems as a hybrid constraint system (HCS), consisting of a flow constraint on trajectories (i.e. continuous functions over time) and a guard constraint on states causing discrete changes. We also propose a technique for solving HCSs by coordinating (i) interval-based solving of nonlinear ODEs, and (ii) a constraint programming technique for reducing interval enclosures of solutions. The proposed technique reliably solves HCSs with nonlinear constraints. Our technique employs the interval Newton method to accelerate the reduction of interval enclosures, while guaranteeing that the enclosure contains a solution.
\end{abstract}

Keywords: Hybrid systems, interval arithmetic, constraint programming

\section{INTRODUCTION}

Detection of states causing discrete changes in continuously evolving hybrid systems plays a significant role in simulation and verification. The problem is described by the conjunction of an ordinary differential equation (ODE) and a condition for a discrete change (guard condition). Many techniques for the problem (e.g. Esposito and Kumar (2007)) perform numerical computation. Since the techniques may compute unexpected results due to rounding errors, various workarounds have been investigated; for example, Park and Barton (1996) handled the discontinuity sticking problem, that is, the problem of detecting the same discrete event right after a discrete change.

Interval arithmetic (Moore et al. (2009)) provides rigor in numerical computation. Computation in interval arithmetic produces over-approximation of continuous states which is a set of intervals or boxes enclosing the theoretical solutions and the accumulation of round-off errors. Interval arithmetic has been applied to the simulation (Nedialkov and von Mohrenschildt (2002)) and verification (Henzinger et al. (2000); Ratschan and She (2007)) of hybrid systems.

Hybrid systems are modeled by constraints, i.e. equations on real numbers and functions, and intervals (inequalities) for uncertain parameters such as initial values (Hickey

* This research is partially supported by JSPS, Grant-in-Aid for Young Scientists (B) 20700033. and Wittenberg (2004)). The reliable simulation and verification are done by integrating continuous dynamics and discrete changes, together with handling uncertainties and computation errors. It is not obvious to reliably compute hybrid systems described by nonlinear ODEs and nonlinear conditions for discrete changes. This paper proposes a framework for such nonlinear hybrid systems.

- We propose hybrid constraint systems (HCSs) to describe the problem of detecting discrete changes by constraints (Section 4). An HCS consists of a flow constraint on trajectories and a guard constraint on continuous states. Solving the HCS is computation of box-consistent domains, which means enclosing states with intervals that satisfy every constraint. We see how an HCS serves as a key component in the simulation of hybrid systems (Section 6.1).

- We develop a technique for solving HCSs by integrating an interval-based method for nonlinear ODEs by Nedialkov et al. (1999), and an interval-based constraint programming framework by van Hentenryck et al. (1997)(Section 5). The technique generates a set of boxes smaller than a specified size that encloses the theoretical solution.

- The proposed technique employs the interval Newton method for the quadratic convergence of the reduction of boxes, and to guarantee that the boxes contain a solution (Section 5.3). The method uses an interval Newton operator derived from flow and guard con- 
straints. Experimental results show the efficiency of the method in simulating nonlinear hybrid systems (Section 6).

\section{RELATED WORK}

This work extends a technique by Ishii et al. (2008). This earlier work was limited in formalizing the problem of detecting discrete changes as a constraint system. The efficiency of the method was also limited since pruning on a time domain was based on a binary search technique.

Park and Barton (1996) introduced the interval Newton method to the detection process to guarantee the existence of an event within a time interval. However, the guarantee is based on interpolation polynomials converted from the original problems. Nedialkov and von Mohrenschildt (2002) proposed an interval-based method for simulating hybrid systems. The method is limited in efficiency and handling of nonlinear guard conditions.

Cruz (2005), Lin and Stadtherr (2007), and others are applying constraint programming techniques to intervalbased solving of ODEs. Their frameworks allow the use of parameters in ODEs as well as the addition of various constraints such as value restriction constraints. Although the frameworks do not handle constraints equivalent to the guard constraints in this paper, the frameworks might be integrated with ours.

\section{PRELIMINARIES}

This section introduces concepts for describing the technique we propose.

\subsection{Interval Arithmetic}

A set of machine-representable floating-point numbers is denoted by $\boldsymbol{F}$. A (bounded) interval $I=[l, u](l, u \in \boldsymbol{F})$ is a set of real numbers, where

$$
I=\{r \in \boldsymbol{R} \mid l \leq r \leq u\} .
$$

$\boldsymbol{I}$ denotes a set of intervals. A box $B$ is a tuple of $n$ intervals $\left(I_{1}, \ldots, I_{n}\right) . \boldsymbol{I}^{n}$ denotes a set of boxes. For an interval $I$, $\mathrm{lb}(I)$ denotes the lower bound, $\mathrm{ub}(I)$ denotes the upper bound, w $(I)$ denotes the width, $\operatorname{int}(I)$ denotes the internal of $I$, and $\mathrm{m}(I)$ denotes the center of $I$. For $r \in \boldsymbol{R},[r]$ denotes the narrowest interval containing $r$.

For $f: \boldsymbol{R}^{m} \rightarrow \boldsymbol{R}^{n}, F: \boldsymbol{I}^{m} \rightarrow \boldsymbol{I}^{n}$ is called an $f^{\prime}$ 's interval extension iff it satisfies the following condition $\left(F_{i}\right.$ denotes the $i$-th component of the value of $F$ )

$$
\begin{gathered}
\forall I_{1} \in \boldsymbol{I} \cdots \forall I_{m} \in \boldsymbol{I} \forall r_{1} \in I_{1} \cdots \forall r_{m} \in I_{m} \forall i \in\{1, \ldots, n\} \\
\left(f_{i}\left(r_{1}, \ldots, r_{m}\right) \in F_{i}\left(I_{1}, \ldots, I_{m}\right)\right) .
\end{gathered}
$$

For $I_{1}, \ldots, I_{m} \in \boldsymbol{I}$ and an interval extension $F$ of $f$, a box $F\left(I_{1}, \ldots, I_{m}\right)$ is called an interval enclosure of possible values of $f$ over $I_{1}, \ldots, I_{m}$. For a bounded set $R \subset \boldsymbol{R}, \square R$ denotes the smallest interval $I \in \boldsymbol{I}$ that encloses $R$. For a constraint $c \subseteq \boldsymbol{R}^{n}, C \subseteq \boldsymbol{I}^{n}$ is an interval extension of $c$ iff it satisfies the following condition

$$
\forall I_{1} \in \boldsymbol{I} \cdots \forall I_{n} \in \boldsymbol{I}
$$

$\left(\forall r_{1} \in I_{1} \cdots \forall r_{n} \in I_{n}\left(\left(r_{1}, \ldots, r_{n}\right) \in c\right) \Rightarrow\left(I_{1}, \ldots, I_{n}\right) \in C\right)$.

\subsection{Interval Newton Method}

Given an equation $h(t)=0$, where $h: \boldsymbol{R} \rightarrow \boldsymbol{R}$ is a continuously differentiable function, a solution of the equation in an interval $T$ is also included in an interval obtained by the following interval operator

$$
N_{H, \dot{H}}(T)=T \cap\left([\mathrm{m}(T)]-\frac{H([\mathrm{~m}(T)])}{\dot{H}(T)}\right),
$$

where $H$ and $\dot{H}$ are interval extensions of $h$ and its derivative. $N_{H, \dot{H}}(T)$ is defined iff $0 \notin \dot{H}(T)$ holds. The (uni-variate) interval Newton method iteratively refines an interval enclosure by the operator above. By taking a sufficiently small enclosure $T$ of a solution, iterated applications of $N_{H, \dot{H}}(T)$ will converge. The fixpoint is denoted by $N_{H, \dot{H}}^{*}(T)$. If the condition $N_{H, \dot{H}}(T) \subseteq \operatorname{int}(T)$ holds, a unique solution $t^{*} \in N_{H, \dot{H}}(T)$ exists (see Theorem 8.4 in Moore et al. (2009)).

\subsection{Interval-based Solving of ODEs}

Let $y$ denotes a vector-valued continuous function over time $\boldsymbol{R} \rightarrow \boldsymbol{R}^{n}$ called trajectory. An initial value problem for an ODE (IVP-ODE) is formed by the conjunction of equations

$$
\dot{y}(t)=f(t, y(t)) \wedge y\left(t_{0}\right)=y_{0},
$$

where $t_{0} \in \boldsymbol{R}, y_{0} \in \boldsymbol{R}^{n}$ and $f: \boldsymbol{R}^{n+1} \rightarrow \boldsymbol{R}^{n}$ (assuming Lipschitz continuity). Given an IVP-ODE, a solution denoted by $y_{t_{0}, y_{0}}$ is a trajectory that satisfies the equations.

Given an initial value set $\left(Y_{0}, T_{0}\right) \in \boldsymbol{I}^{n+1}$, an interval extension of the solution $y_{t_{0}, y_{0}}$, denoted by $Y_{T_{0}, Y_{0}}: \boldsymbol{I} \rightarrow$ $\boldsymbol{I}^{n}$, satisfies the following condition

$$
\forall t_{0} \in T_{0} \forall y_{0} \in Y_{0} \forall t \in T\left(y_{t_{0}, y_{0}}(t) \in Y_{T_{0}, Y_{0}}(T)\right),
$$

where $T$ is a time interval such that $\mathrm{lb}(T) \geq \mathrm{ub}\left(T_{0}\right)$.

We employ an existing method VNODE proposed in Nedialkov et al. (1999) and Nedialkov (2006) for solving IVP-ODEs based on interval arithmetic. Consider an IVPODE, an initial value set $\left(T_{0}, Y_{0}\right)$ and a time interval $T_{1} \in \boldsymbol{I}$. We obtain a box $Y_{1}=Y_{T_{0}, Y_{0}}\left(T_{1}\right)$ using VNODE. As a by-product of the computation, VNODE computes an enclosure $Y_{T_{0}, Y_{0}}\left(\left[\mathrm{lb}\left(T_{0}\right), \mathrm{ub}\left(T_{1}\right)\right]\right)$, because the computation is done iteratively from the initial value.

In our method, we also need an interval extension of the derivative of the solution $\dot{Y}_{T_{0}, Y_{0}}: \boldsymbol{I} \rightarrow \boldsymbol{I}^{n}$. Let $T_{1} \in \boldsymbol{I}$ be a time interval. Then $\dot{Y}_{T_{0}, Y_{0}}\left(T_{1}\right)$ will be implemented as computation of a function $F\left(T_{1}, Y_{T_{0}, Y_{0}}\left(T_{1}\right)\right)$, that is, an interval extension of $f(t, y(t))$ in the ODE. Note that $Y_{T_{0}, Y_{0}}\left(T_{1}\right)$ should be computed by VNODE beforehand.

\subsection{Consistency Technique for Continuous Constraints}

Van Hentenryck et al.(1997) proposed a method for solving a set of continuous constraints $\mathcal{C}$ with a tuple of $n$ variables $\mathcal{X}=\left(x_{1}, \ldots, x_{n}\right)$ and a domain $\mathcal{D}=\left(D_{1}, \ldots, D_{n}\right)$. See Benhamou and Granvilliers (2006) for an introduction. $\mathcal{C}$ is a conjunction of constraints in the form of $g(\mathcal{X}) \bullet 0$, where - is a relation e.g. $\leq$ and $=$, and $g$ is a continuous function $\boldsymbol{R}^{n} \rightarrow \boldsymbol{R}$ built with elementary arithmetic operations. The method refines a given domain $\mathcal{D}$ by the BRANCHANDPRUNE algorithm and computes a set of boxes smaller 
Input: set of constraints $\mathcal{C}$, initial domain $\mathcal{D}$, error tolerance $\epsilon$

Output: set of consistent domains $\left\{\mathcal{D}_{i}\right\}_{i \in\{1, \ldots, n\}}$

1: $\quad \mathcal{D}^{\prime}:=\operatorname{PrunE}(\mathcal{C}, \mathcal{D})$

2: $\quad$ if $\mathcal{D}^{\prime} \neq \emptyset$ then

3: $\quad$ if the precision of $\mathcal{D}^{\prime}$ is under $\epsilon$ then

4: $\quad$ return $\left\{\mathcal{D}^{\prime}\right\}$

5: $\quad$ else

6: $\quad i:=$ select a component

7: $\quad\left(\mathcal{D}_{1}, \mathcal{D}_{2}\right):=\operatorname{BRANCH}\left(\mathcal{D}^{\prime}, i\right)$

8: $\quad$ return $\operatorname{BranCHANdPRUNe}\left(\mathcal{C}, \mathcal{D}_{1}\right) \cup$

BranchandPrune $\left(\mathcal{C}, \mathcal{D}_{2}\right)$

$9:$

10:

11:

12 :

end if

else

return $\emptyset$

end if

Fig. 1. BRANCHANDPRUNE algorithm.

than a given error tolerance $\epsilon$, so that the union of boxes is a box-consistent domain (see Section 4.1) for the constraint system. Figure 1 shows the BRANCHANDPRUNE algorithm. The algorithm recursively alternates reducing and branching of a domain in a problem. In the algorithm, two procedures are left open as PRUNE and BRANCH. Algorithms such as HC4, BC5 and 3B are known as implementations for PRUNE. BRANCH is usually implemented as partitioning of a domain into several boxes along a dimension of the domain.

\section{HYBRID CONSTRAINT SYSTEMS}

In this section, we define HCSs. We consider continuous states as $(n+1)$ dimensional real vectors over time and space. An HCS describes a crossing point of a trajectory over time and a time-invariant boundary in the state space. Figure 2 illustrates an HCS.

Consider a tuple of variables $\mathcal{X}=\left(x_{0}, x_{1}, \ldots, x_{n}\right)$ consisting of a variable $x_{0}$ representing the time at a crossing point and $n$ variables $x_{1}, \ldots, x_{n}$ representing a state at the time $x_{0}$. We then define the following two kinds of constraints.

- A flow constraint $\mathcal{C}_{f}$ is a constraint described by the conjunction of the following equations

$$
\begin{aligned}
(\dot{y}(t)=f(t, y(t)) & \left.\wedge y\left(t_{0}\right)=y_{0}\right) \\
& \wedge y\left(x_{0}\right)=\left(x_{1}, \ldots, x_{n}\right) \wedge x_{0}>t_{0},
\end{aligned}
$$

where the first part is an IVP-ODE representing an $n$-dimensional trajectory $y_{t_{0}, y_{0}}\left(t_{0} \in \boldsymbol{R}, y_{0} \in \boldsymbol{R}^{n}\right.$, and $f: \boldsymbol{R}^{n+1} \rightarrow \boldsymbol{R}^{n}$ is a Lipschitz continuous function).

- A guard constraint $\mathcal{C}_{g}$ is a constraint described by

$$
g\left(x_{1}, \ldots, x_{n}\right)=0,
$$

where $g$ is a differentiable function $\boldsymbol{R}^{n} \rightarrow \boldsymbol{R}$.

Possible values for the variables belong to a domain $\mathcal{D}=\left(D_{0}, D_{1}, \ldots, D_{n}\right) \in \boldsymbol{I}^{n+1}$, where each component is associated with a variable in $\mathcal{X}$. Values expressing parameters $t_{0}$ and $y_{0}$ in the initial condition in $\mathcal{C}_{f}$ are given as an initial value set $\mathcal{D}_{0}=\left(D_{0,0}, \ldots, D_{n, 0}\right) \in \boldsymbol{I}^{n+1}$. A hybrid constraint system is a tuple $\left(\mathcal{X}, \mathcal{D}, \mathcal{D}_{0}, \mathcal{C}_{f}, \mathcal{C}_{g}\right)$ with a tuple $\mathcal{X}$ of variables, a domain $\mathcal{D}$, an initial value set $\mathcal{D}_{0}$, a flow constraint $\mathcal{C}_{f}$ and a guard constraint $\mathcal{C}_{g}$.
For example, a ball that bounces off a sinusoidal surface is modeled as an $\operatorname{HCS}\left(\mathcal{X}, \mathcal{D}, \mathcal{D}_{0}, \mathcal{C}_{f}, \mathcal{C}_{g}\right)$, where

$$
\begin{aligned}
& \mathcal{X}=\left(x_{0}, x_{1}, x_{2}, x_{3}, x_{4}\right), \\
& \mathcal{D}=\left(\boldsymbol{R}^{\geq 0}, \boldsymbol{R}, \boldsymbol{R}, \boldsymbol{R}, \boldsymbol{R}\right), \mathcal{D}_{0}=([0],[1],[3],[6],[-2]), \\
& \mathcal{C}_{f} \equiv\left(\dot{y}(t)=\left(\dot{y}_{1}(t), \dot{y}_{2}(t), \dot{y}_{3}(t), \dot{y}_{4}(t)\right)\right. \\
&\left.=\left(y_{3}(t), y_{4}(t), 0,-g-k \cdot y_{4}(t)\right) \wedge y\left(t_{0}\right)=y_{0}\right) \\
& \wedge y\left(x_{0}\right)=\left(x_{1}, x_{2}, x_{3}, x_{4}\right) \wedge x_{0}>t_{0}, \\
& \mathcal{C}_{g} \equiv \sin \left(2 \cdot x_{1}\right)-x_{2}=0 .
\end{aligned}
$$

$x_{0}$ is a variable representing time, $x_{1}, x_{2}, x_{3}$ and $x_{4}$ are variables representing the position $\left(x_{1}, x_{2}\right)$ and velocity $\left(x_{3}, x_{4}\right)$ of the ball. Movement of the ball is described by $\mathcal{C}_{f}$ and a contact with the surface is detected by checking whether $\mathcal{C}_{g}$ is entailed. The initial condition in $\mathcal{C}_{f}$ is interpreted as $\forall t_{0} \in[0] \forall y_{0} \in[1] \times[3] \times[6] \times[-2]\left(y\left(t_{0}\right)=y_{0}\right)$. Figure 2 (a) illustrates the trajectory of the ball with parameters set to $g=9.8$ and $k=0.3$.

Let $\left(\mathcal{X}, \mathcal{D}, \mathcal{D}_{0}, \mathcal{C}_{f}, \mathcal{C}_{g}\right)$ is an HCS, a valuation is a map $\mathcal{X} \rightarrow \mathcal{D}$ from every variable $x_{i} \in \mathcal{X}$ to a value $d_{i} \in D_{i}$ $(i \in\{0, \ldots, n\})$. A solution of the HCS is a valuation satisfying constraints $\mathcal{C}_{f}$ and $\mathcal{C}_{g}$. In general, an HCS may have multiple solutions. When applying HCSs to the simulation of hybrid systems, the one we are interested in is the earliest solution. In Figure 2 (a), the HCS has three solutions inside each of the boxes $\mathcal{D}_{1}, \mathcal{D}_{2}$ and $\mathcal{D}_{3}$. The ball bounces at the earliest solution in $\mathcal{D}_{1}$.

\subsection{Box Consistency for HCSs}

In this paper, interval-based solving of an HCS means refining an initial domain to a box-consistent (van Hentenryck et al. (1997)) domain.

We modify the definition of box consistency for HCSs as follows. Let $y$ be a solution trajectory of a flow constraint and $g$ be a function describing a guard constraint. Consider interval extensions $G$ of $g$ and $Y_{T_{0}, Y_{0}}$ of $y_{t_{0}, y_{0}}$, where $T_{0}$ is $D_{0,0}$ and $Y_{0}$ is $\left(D_{1,0}, \ldots, D_{n, 0}\right)$. For an index $i \in$ $\{0, \ldots, n\}$, the $i$-th component $D_{i}$ of a domain $\mathcal{D}$ is boxconsistent with respect to the other components iff

$$
D_{0}=\square\left\{d_{0} \in D_{0} \mid 0 \in G\left(Y_{T_{0}, Y_{0}}\left(\left[d_{0}\right] \pm\left[0, h_{m i n}\right]\right)\right)\right\}
$$

(for $i=0$ ), where $h_{\text {min }} \in \boldsymbol{R}^{+}$and $h_{\text {min }} \geq \mathrm{w}\left(T_{0}\right)$, and

$$
\begin{aligned}
& D_{i}=\square\left\{d_{i} \in D_{i} \mid d_{i} \in Y_{T_{0}, Y_{0}, i}\left(D_{0}\right) \wedge\right. \\
& \quad 0\left.\in G\left(D_{1}, \ldots, D_{i-1},\left[d_{i}\right], D_{i+1}, \ldots, D_{n}\right)\right\}
\end{aligned}
$$

(for $i \in\{1, \ldots, n\}$ ). An HCS is box-consistent iff all the components are box-consistent. $h_{\text {min }}$ in the condition above is used because it is difficult to compute $Y_{T_{0}, Y_{0}}\left(\left[d_{0}\right]\right)$ by VNODE.

Consider a set of floating-point numbers, $\boldsymbol{F}=\{n$. $\left.10^{-2} \mid n \in \boldsymbol{Z}\right\}$. For the bouncing ball example, a box $\mathcal{D}_{1}=([0.44,0.54],[3.86,3.96],[0.95,1.05],[5.95,6.05]$, $[-6.1,-6.2])$ is appropriate for the box-consistent domain.

\section{TECHNIQUE FOR SOLVING AN HCS}

In this section, a technique for computing the boxconsistent domain of an HCS is described. The technique applies the BRANCHANDPRUNE algorithm in Section 3.4 to HCSs with an implementation of PRUNE called PRUNEHCS. The proposed method computes a set of boxes that encloses all the solutions in the initial domain. The 


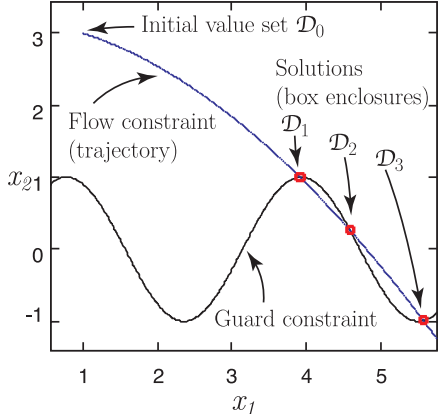

(a)

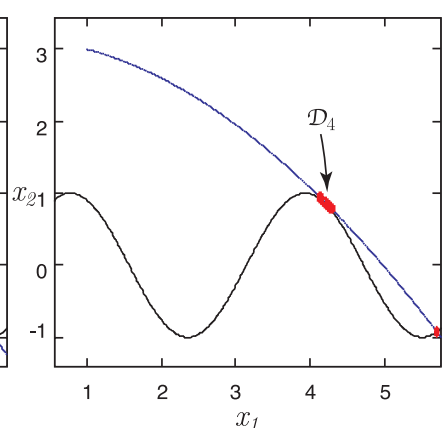

(b)
Fig. 2. Examples of an HCS.

Input: flow constraint $\mathcal{C}_{f} \equiv(\dot{y}(t)=f(t, y(t)) \wedge$ $\left.y\left(t_{0}\right)=y_{0}\right) \wedge\left(x_{1}, \ldots, x_{n}\right)=y\left(x_{0}\right) \wedge x_{0}>t_{0}$, guard constraint $\mathcal{C}_{g} \equiv g\left(x_{1}, \ldots, x_{n}\right)=0$, initial domain $\mathcal{D}=\left(D_{0}, D_{1}, \ldots, D_{n}\right) \in \boldsymbol{I}^{n+1}$, initial value set $\mathcal{D}_{0}$

Output: box-consistent domain $\mathcal{D}^{\prime}=\left(D_{0}^{\prime}, \ldots, D_{n}^{\prime}\right) \in \boldsymbol{I}^{n+1}$ 1: repeat

2: $\quad \mathcal{D}^{\prime}:=\mathcal{D}$

3: $\quad$ obtain $H$ and $\dot{H}$ from $\mathcal{C}_{g}, \mathcal{C}_{f}$ and $\mathcal{D}_{0}$

4: $\quad D_{0}:=\operatorname{NarrowL}\left(H, \dot{H}, D_{0}\right)$

5: $\quad D_{0}:=\operatorname{NarrowU}\left(H, \dot{H}, D_{0}\right)$

6: $\quad\left(D_{1}, \ldots, D_{n}\right):=\left(D_{1}, \ldots, D_{n}\right) \cap Y_{T_{0}, Y_{0}}\left(D_{0}\right)$

7: $\quad\left(D_{1}, \ldots, D_{n}\right):=\operatorname{NaRrow}_{\mathrm{CCS}}\left(\mathcal{C}_{g},\left(D_{1}, \ldots, D_{n}\right)\right)$

8: $\quad$ until $\mathcal{D}=\mathcal{D}^{\prime}$

9: $\quad$ return $\mathcal{D}^{\prime}$

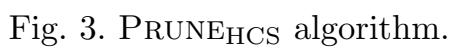

accuracy of results are specified by the real-valued parameters $\epsilon$ and $h_{m i n} . \epsilon$ used in the BRANCHANDPRUNE algorithm determines the maximum width of intervals in a result. A solution exists within $h_{\text {min }}$ from each bound of the time domain.

Figure 3 shows the PRUNE HCS $_{\text {algorithm. PRUNE }}$ PCS reduces a domain of an HCS by enforcing the box consistency. PRUNEHCS takes a flow constraint $\mathcal{C}_{f}$, a guard constraint $\mathcal{C}_{g}$, a domain $\mathcal{D}$, and an initial value set $\mathcal{D}_{0}$ as input. PRUNEHCS iteratively prunes each component of a domain until the fixpoint is reached (lines 1-8).

At lines $3-5$, the domain $D_{0}$ of the time variable $x_{0}$ is reduced, and then at lines $6-7$, the domain $\left(D_{1}, \ldots, D_{n}\right)$ is reduced. Details on each of the reduction methods are supplied in Sections 5.1 and 5.2. The algorithm returns a pruned domain $\mathcal{D}^{\prime}$ at line 9 .

\subsection{Reduction of the Time Domain}

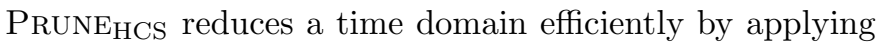
the interval Newton method that solves flow and guard constraints simultaneously. At line 3 in Figure 3, the algorithm constructs functions $H$ and $\dot{H}$ for the interval Newton method. The essential idea is that the numerical solution of the IVP-ODE, denoted hereafter as $Y_{T_{0}, Y_{0}}$, is used to construct an interval Newton operator. $Y_{T_{0}, Y_{0}}$ is obtained by iterative calculation with respect to a flow constraint $\mathcal{C}_{f}$ and an initial value set $\mathcal{D}_{0}$, as described in Section 3.3. Accordingly, $H$ and $\dot{H}$ are given by
$H(T)=G\left(Y_{T_{0}, Y_{0}}(T)\right), \dot{H}(T)=\sum_{i=1}^{n}\left(\frac{\partial G}{\partial X_{i}} \cdot \dot{Y}_{T_{0}, Y_{0}, i}(T)\right)$,

where $Y_{T_{0}, Y_{0}}, G, \dot{Y}_{T_{0}, Y_{0}}$ and $\partial G / \partial X_{i}$ are interval extensions of a trajectory $y_{t_{0}, y_{0}}$, a function $g$ in a guard constraint $\mathcal{C}_{g}$, and their derivatives, respectively. $X_{i}$ is a variable of $G$ $(i \in\{1, \ldots, n\})$. To compute $\partial G / \partial X_{i}$, we apply automatic differentiation to $G$.

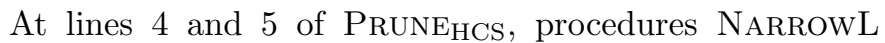
and NARROWU reduce the lower and upper edges of the domain. This procedure is an adaptation to HCS of the algorithm shown in van Hentenryck et al. (1997). The NARROWL procedure is as follows (NARROwU is similar except that it operates on the upper edge instead of the lower edge).

(1) First, the guard constraint is checked for the current time domain $D_{0}$. If $0 \notin H\left(D_{0}\right)$ holds, return $\emptyset$ and terminate.

(2) Calculate the fixpoint of the interval Newton method $D_{0}^{\prime}=N_{H, \dot{H}}^{*}\left(D_{0}\right)$. To obtain $N_{H, \dot{H}}^{*}\left(D_{0}\right), D_{0}^{\prime}=$ $N_{H, \dot{H}}\left(D_{0}\right)$ is repeatedly computed until the ratio of $D_{0}^{\prime}$ to $D_{0}$ is under a threshold.

(3) Let $h_{\text {min }}$ is a minimal step width for ODE solving, and consider the box $L=\left[\operatorname{lb}\left(D_{0}^{\prime}\right), \operatorname{lb}\left(D_{0}^{\prime}\right)+h_{\text {min }}\right]$. If the interval $H(L)$ contains 0 , return an interval $\left[\operatorname{lb}\left(D_{0}^{\prime}\right)\right.$, $\left.\mathrm{ub}\left(D_{0}\right)\right]$, and terminate.

(4) Split $D_{0}$ in two and apply NARRowL recursively for each interval. Find the smallest bound $l$ in the results, return an interval $\left[l, \mathrm{ub}\left(D_{0}\right)\right]$, and terminate.

\subsection{Reduction of the Continuous State Domain}

Once the narrowing operators reduce a time interval $D_{0}$, VNODE computes a box $Y_{T_{0}, Y_{0}}\left(D_{0}\right)$ enclosing the trajectories over $D_{0}$ as described in Section 3.3 (line 6 of Figure 3). Then we can build a continuous constraint system described in Section 3.4 consisting of the guard constraint $\mathcal{C}_{g} \equiv g\left(x_{1}, \ldots, x_{n}\right)=0$ and the initial domain $\left(D_{1}, \ldots, D_{n}\right)=Y_{T_{0}, Y_{0}}\left(D_{0}\right)$. At line 7 of PRUNE PCS $_{\text {, the }}$ NARROW $_{C C S}$ procedure reduces the domain $\left(D_{1}, \ldots, D_{n}\right)$ by applying one of the narrowing operators shown in Section 3.4.

\subsection{Testing the Unique Existence of a Solution}

In general, the number of solutions in boxes computed by BRANCHANDPRUNE is unknown. Using the interval Newton method, the uniqueness and existence of a solution within a box is determined under a certain condition.

Theorem 1. Consider an $\operatorname{HCS}\left(\mathcal{X}, \mathcal{D}, \mathcal{D}_{0}, \mathcal{C}_{f}, \mathcal{C}_{g}\right)$, where $\mathcal{D}=\left(D_{0}, \ldots, D_{n}\right)$. Suppose a call to the procedure PRUNE $_{\text {HCS }}\left(\mathcal{C}_{f}, \mathcal{C}_{g}, \mathcal{D}, \mathcal{D}_{0}\right)$ returns $\mathcal{D}^{\prime}=\left(D_{0}^{\prime}, \ldots, D_{n}^{\prime}\right)$. In the computation, suppose the procedure NARROWL or NARrowU reduces a time interval $D_{0}^{\prime \prime}$ to $N_{H, \dot{H}}\left(D_{0}^{\prime \prime}\right)$, and the following conditions hold. Then, a solution of the HCS uniquely exists in $\mathcal{D}^{\prime}$.

- A unique trajectory $y_{t_{0}, y_{0}}$ exists over $D_{0}$ with respect to $\mathcal{C}_{f}$ and $\mathcal{D}_{0}$,

- $g$ in $\mathcal{C}_{g}$ is continuously differentiable over $\left(D_{1}, \ldots, D_{n}\right)$,

- $N_{H, \dot{H}}\left(D_{0}^{\prime \prime}\right) \subseteq \operatorname{int}\left(D_{0}^{\prime \prime}\right)$,

- $D_{0}^{\prime} \subseteq D_{0}^{\prime \prime}$. 
Proof. From the first and second conditions, a function $g \circ y_{t_{0}, y_{0}}$ exists, and is continuously differentiable over $D_{0}$. Since $G \circ Y_{T_{0}, Y_{0}}$ composed by PRUNE $_{H C S}$ is an interval extension of $g \circ y_{t_{0}, y_{0}}$, the interval Newton method proves that a root of $g \circ y_{t_{0}, y_{0}}$ uniquely exists in $D_{0}^{\prime \prime}$. The third condition is for the interval Newton method. The last condition proves the unique existence of the solution in $\mathcal{D}^{\prime}$ because the computation by PRUNE $_{\text {HCS }}$ completely encloses the solution.

VNODE validates the first condition for the computed enclosure. The third condition will be tested in the narrowing procedures.

For example, the box-consistent domain for the bouncing ball shown in the previous section encloses a unique solution. Consider a bouncing ball with an initial value set $\mathcal{D}_{0}=([0],[1],[3],[6],[-1.7,-1.6])$ (see Figure $2(\mathrm{~b})$ ). The box-consistent domain $\mathcal{D}_{4}$ indicated in the figure is not guaranteed to have a solution.

\subsection{Computing an Enclosure for the Earliest Solution}

The union of boxes computed by the proposed technique may enclose multiple solutions. Boxes enclosing the earliest solution are selected as follows.

(1) Compute the clusters of boxes by concatenating two boxes if they are adjacent.

(2) Find a cluster containing the earliest time.

(3) If the cluster intersects with the initial value set, then discard this and search for the next earliest cluster.

Note that we assume each of the clusters computed in (1) and the initial value set in (3) enclose a unique solution.

\section{EXAMPLES AND EXPERIMENTATION}

We implemented the proposed method on top of the Elisa system (Granvilliers and Sorin (2005)) which is an implementation of the BRANCHANDPRUNE algorithm. We implemented data structures for representing flow constraints. PRUNEHCS was implemented by extending the reduction procedure in Elisa. VNODE-LP (Nedialkov (2006)) was used to solve IVP-ODEs. The implementation caches the results by VNODE-LP for reusing. Our implementation consists of about 2000 lines of $\mathrm{C}++$ code.

Section 6.1 shows a simulation of a bouncing ball by modeling each bounce of the ball as an HCS. Section 6.2 reports the results of comparisons with existing methods. The parameters in the proposed method were set as $\epsilon=$ $10^{-2}$ and $h_{\min }=10^{-11}$. BC5 was used as NARROW NCS $_{\text {. }}$ In the experimentation, we modified the implementation to terminate the computation after an enclosure for the earliest solution was obtained. We experimented on a $2.4 \mathrm{GHz}$ Intel Core 2 Duo processor with $2 \mathrm{~GB}$ of RAM.

\subsection{Computation of a Bouncing Ball}

Consider the bouncing ball described in Section 4 . Here, we change the initial value set to $\mathcal{D}_{0}=([0],[2],[5],[0],[-5])$, and the guard constraint to $\mathcal{C}_{g} \equiv \sin \left(x_{1}\right)-x_{2}=0$. Figure 4 illustrates the boxes enclosing a trajectory of the ball bouncing off the surface three times. Those boxes

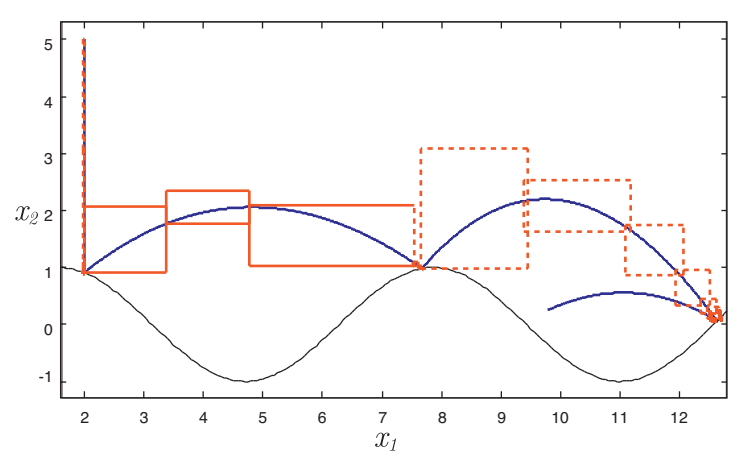

Fig. 4. A trajectory of a bouncing ball on a sinusoidal surface and an interval enclosure of the trajectory.

were computed while solving three HCSs, each of which corresponded to a parabolic motion of the ball. From the result of solving an HCS, a bounce of the ball with a coefficient of restitution of 0.8 was computed by interval arithmetic to set up the initial values for the next phase.

We confirmed that the interval $D_{0}$ of the domain was quadratically reduced by the interval Newton operator. For example, in the solving of the first bounce, we had

$$
\begin{aligned}
D_{0}^{0} & =[0 . \underline{5} 366024006905468,0.6462371408659776], \\
D_{0}^{1} & =[0 . \underline{56} 55469230670141,0 . \underline{5675738062165443}], \\
D_{0}^{2} & =[0 . \underline{56636} 29650559695,0 . \underline{5663632653947441}], \\
D_{0}^{3} & =[0 . \underline{56636310070488} 00,0 . \underline{5663631007048839}] .
\end{aligned}
$$

The reductions provide the guarantee of the existence and uniqueness of a solution within the domain.

The left half of Table 1 shows computation results. Each row corresponds to a result of solving an HCS. Each column shows the number of bounces, the time interval, the number of calling PRUNE ${ }_{H C S}$, the number of calling NARrowL and NARrowU, and the execution time, in milliseconds, of a solving. The width of the first result was $10^{-11}$, according to the value of $h_{\min }$. The following results widened as the initial value set widened.

\subsection{Comparison with Existing Methods}

To evaluate the computational efficiency and to confirm the accuracy of the results, we solved several HCS problems with several solvers including the proposed method.

The following problems were solved: $(1,2)$ the first and second bounces of the bouncing ball; (3) the problem of detecting the intersection of a trajectory following the Van der Pol equation

$\dot{y}_{1}=y_{2}, \dot{y}_{2}=10 \cdot\left(1-y_{1}^{2}\right) \cdot y_{2}-y_{1}, y_{1}(0)=1, y_{2}(0)=0$, and the ellipse $\mathcal{C}_{g} \equiv x_{1}^{2} / 9+x_{2}^{2} / 255=1$ (we abbreviate $y_{i}(t)$ with $y_{i}$ ); (4) detection of the intersection of a trajectory following the Lorenz equation

$$
\begin{gathered}
\dot{y}_{1}=10 \cdot\left(y_{2}-y_{1}\right), \dot{y}_{2}=y_{1} \cdot\left(28-y_{3}\right)-y_{2}, \\
\dot{y}_{3}=y_{1} \cdot y_{2}-8 / 3 \cdot y_{3}, y_{1}(0)=15, y_{2}(0)=15, y_{3}(0)=36,
\end{gathered}
$$
and the sphere $\mathcal{C}_{g} \equiv x_{1}^{2}+x_{2}^{2}+\left(x_{3}-28\right)^{2}=700$. We solved the above problems with the following methods: (a) the proposed method; (b) the method proposed by Ishii et al. (2008) that employs a binary search technique; (c) the symbolic DSolve solver with the Minimize function in Mathematica 6.0 (Wolfram Research (2007)); and (d, d') 
Table 1. Computation results for the bouncing ball model.

\begin{tabular}{r|l|r|r|r|l|r|r|r}
\hline \hline & \multicolumn{9}{|c|}{ (a) proposed method } & \multicolumn{4}{c}{ (b) existing method } \\
$n$ & result $\left(D_{0}\right)$ & 1 & 2 & 61 & $0.566363100[662,748]$ & 3 & 132 & 515 \\
\hline 1 & $0.5663631007[04,14]$ & 5 & 27 & 171 & $1.51931342[1229,1508]$ & 5 & 210 & 734 \\
2 & $1.5193134214[00,25]$ & 5 & 19 & 125 & $2.6883363[04386,10239]$ & 5 & 200 & 718 \\
3 & $2.688336307[167,706]$ & 5 & branch & reduce & time & result $\left(D_{0}\right)$ & reduce & time \\
\hline
\end{tabular}

Table 2. Comparison results.

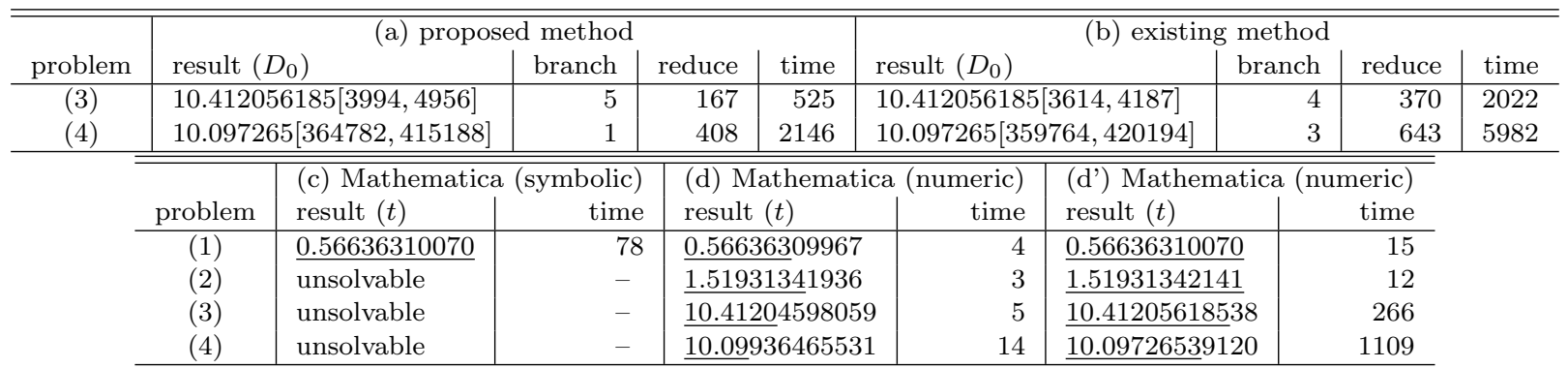

the numerical NDSolve solver with the EventLocator option in Mathematica 6.0. We solved the problem with the default settings in (d), and by setting WorkingPrecision to 28 and MaxSteps to Infinity in (d') ${ }^{1}$.

Table 1 and Table 2 report the computed (interval) values for the time variable $x_{0}$ (represented by $D_{0}$ and $t$ ), profiling results, and execution time in milliseconds. As shown in the results (a) and (b), the proposed technique decreases the number of reductions and outperforms the method (b) in efficiency. In (c), DSolve of Mathematica computed a rigorous solution but treated only the problem (1). The results (d) and (d') show that NDSolve solved HCSs more efficiently than our method. However, NDSolve uses approximation algorithms and cannot ensure the achieved accuracy of a result, whereas our method guarantees the accuracy of a result. Another advantage of our method is that we can give intervals to the initial values and coefficients in constraints. DSolve and NDSolve do not handle ODEs with uncertain parameters.

\section{CONCLUSION AND FUTURE WORK}

We have presented HCSs and proposed a technique for solving them. As described in Section 5.1, the technique guarantees the existence and uniqueness of a solution in a domain. This method also helps to find the earliest solutions reliably. We need more experimentation in realistic settings and various optimization of the implementation. HCSs will be extended to have multiple guard constraints either in conjunctive and disjunctive way. We plan to solve such problems by interacting with SAT solvers to compute every combination of constraints.

\section{REFERENCES}

Benhamou, F. and Granvilliers, L. (2006). Continuous and interval constraints. Handbook of Constraint Programming, 571-604. Elsevier.

Cruz, J. (2005). Constraint Reasoning for Differential Models. IOS Press.

1 The corresponding Mathematica notebooks are available at http://www . ueda.info.waseda.ac.jp/ ishii/pub/adhs2009/.
Esposito, J. and Kumar, V. (2007). A state event detection algorithm for numerically simulating hybrid systems with model singularities. In $A C M$ TOMACS, 17(1), 122 .

Granvilliers, L. and Sorin, V. (2005). Elisa 1.0.4. http://sourceforge.net/projects/elisa.

Henzinger, T.A., Horowitz, B., Majumdar, R., and WongToi, H. (2000). Beyond HyTech: hybrid systems analysis using interval numerical methods. In Proc. $H S C C$, LNCS 1790, 130-144.

Hickey, T.J. and Wittenberg, D.K. (2004). Rigorous modeling of hybrid systems using interval arithmetic constraints. In Proc. HSCC, LNCS 2993, 402-416.

Ishii, D., Ueda, K., and Hosobe, H. (2008). An intervalbased consistency technique for reliable simulation of hybrid systems. In IPSJ Trans. on Mathematical Modeling and its Applications, 1(1), 149-159. (in Japanese)

Lin, Y. and Stadtherr, M. (2007). Fault detection in continuous-time systems with uncertain parameters. In Proc. ACC, 3216-3221.

Moore, R.E., Kearfott, R.B., and Cloud, M.J. (2009). Introduction to Interval Analysis. SIAM.

Nedialkov, N.S. (2006). VNODE-LP: a validated solver for initial value problems in ordinary differential equations. TR CAS-06-06-NN, McMaster University.

Nedialkov, N.S., Jackson, K.R., and Corliss, G.F. (1999). Validated solutions of initial value problems for ordinary differential equations. In Applied Mathematics and Computation, 105(1), 21-68.

Nedialkov, N.S. and von Mohrenschildt, M. (2002). Rigorous simulation of hybrid dynamic systems with symbolic and interval methods. In Proc. ACC, vol. 1, 140-147.

Park, T. and Barton, P. (1996). State event location in differential-algebraic models. In ACM TOMACS, 6(2), $137-165$.

Ratschan, S. and She, Z. (2007). Safety verification of hybrid systems by constraint propagation-based abstraction refinement. In $A C M$ TECS, 6(1).

van Hentenryck, P., McAllester, D., and Kapur, D. (1997). Solving polynomial systems using a branch and prune approach. In SIAM Journal on Numerical Analysis, $34(2), 797-827$.

Wolfram Research (2007). Mathematica 6.0. http://www.wolfram.com/products/mathematica. 